\title{
Effect of Enhanced Primary Care for People with Serious Mental IIIness on Service Use and Screening
}

\author{
Lexie R. Grove, $\mathrm{MSPH}^{7}{ }^{\oplus}$, Alex K. Gertner, $P h D^{7}$, Karen E. Swietek, PhD, MPH', \\ Ching-Ching Claire Lin, PhD', Neepa Ray, MS ${ }^{3}$, Tyler L. Malone, MS ${ }^{7}$, \\ David L. Rosen, MD, PhD', Theodore R. Zarzar, MD ${ }^{5}$, Marisa Elena Domino, PhD 1,5,6, \\ Brian Sheitman, $M D^{7}$, and Beat D. Steiner, $M D, M P H^{8}$
}

'Department of Health Policy and Management, Gillings School of Global Public Health, University of North Carolina at Chapel Hill, 135 Dauer Dr., Chapel Hill, NC, USA; ${ }^{2}$ NORC at the University of Chicago, Boston, USA; ${ }^{3}$ Center for Medication Optimization, Eshelman School of Pharmacy, University of North Carolina at Chapel Hill, Chapel Hill, USA; ${ }^{4}$ Institute for Global Health and Infectious Diseases, Division of Infectious Diseases, School of Medicine, University of North Carolina at Chapel Hill, Chapel Hill, USA; ${ }^{5}$ Department of Psychiatry, School of Medicine, University of North Carolina at Chapel Hill, Chapel Hill, USA; ${ }^{6}$ Cecil G. Sheps Center for Health Services Research, University of North Carolina at Chapel Hill, Chapel Hill, USA; ${ }^{7}$ North Carolina Department of Public Safety-Prisons, Raleigh, USA; ${ }^{8}$ Department of Family Medicine, School of Medicine, University of North Carolina at Chapel Hill, Chapel Hill, USA; ${ }^{9}$ National Taiwan University, Taipei, Taiwan.

BACKGROUND: Strategies are needed to better address the physical health needs of people with serious mental illness (SMI). Enhanced primary care for people with SMI has the potential to improve care of people with SMI, but evidence is lacking.

OBJECTIVE: To examine the effect of a novel enhanced primary care model for people with SMI on service use and screening.

DESIGN: Using North Carolina Medicaid claims data, we performed a retrospective cohort analysis comparing healthcare use and screening receipt of people with SMI newly receiving enhanced primary care to people with SMI newly receiving usual primary care. We used inverse probability of treatment weighting to estimate average differences in outcomes between the treatment and comparison groups adjusting for observed baseline characteristics.

PARTICIPANTS: People with SMI newly receiving primary care in North Carolina.

INTERVENTIONS: Enhanced primary care that includes features tailored for individuals with SMI.

MAIN MEASURES: Outcome measures included outpatient visits, emergency department (ED) visits, inpatient stays and days, and recommended screenings 18 months after the initial primary care visit.

KEY RESULTS: Compared to usual primary care, enhanced primary care was associated with an increase of 1.2 primary care visits $(95 \%$ confidence interval [CI]: 0.31 to 2.1 ) in the 18 months after the initial visit and decreases of 0.33 non-psychiatric inpatient stays (CI: -0.49 to -0.16 ) and 3.0 non-psychiatric inpatient days (CI: -5.3 to -0.60 ). Enhanced primary care had no significant effect on psychiatric service and ED use. Enhanced primary care increased the probability of glucose and HIV screening, decreased the probability of lipid screening, and had no effect on hemoglobin Alc and colorectal cancer screening.

Prior Presentations N/A

Received April 21, 2020

Accepted December 9, 2020

Published online January27, 2021
CONCLUSIONS: Enhanced primary care for people with SMI can increase receipt of some preventive screening and decrease use of non-psychiatric inpatient care compared to usual primary care.

KEY WORDS: integrated care; enhanced primary care; serious mental illness; schizophrenia; Medicaid.

J Gen Intern Med 36(4):970-7

DOI: $10.1007 / \mathrm{s} 11606-020-06429-2$

(c) Society of General Internal Medicine 2021

\section{INTRODUCTION}

Effectively addressing the health needs of the 11.4 million adults living with serious mental illness (SMI) is one of the major challenges facing the US' healthcare system. ${ }^{1} \mathrm{SMI}$ is defined as any mental illness that results in serious functional impairment. ${ }^{2}$ Adults with SMI have a higher prevalence of chronic physical health conditions than the general population, ${ }^{3-5}$ receive lower quality care for these conditions, ${ }^{6-9}$ and experience worse physical health outcomes that drive highcost healthcare use. ${ }^{10-13}$ Better addressing the physical health needs of people with SMI could have substantial public health effects. People with SMI die an average of 25 years earlier than people without SMI, and most of these premature deaths are from treatable physical health conditions, including cardiovascular, pulmonary, and infectious diseases. ${ }^{14}$

Integrated care models that address physical and mental health, such as behavioral health homes (BHHs) and patientcentered medical homes (PCMHs), hold promise for improving the care of this population. The $\mathrm{BHH}$ model integrates primary care services in behavioral health $(\mathrm{BH})$ clinic settings. ${ }^{15}$ Existing studies suggest that BHHs may improve preventive screening, increase primary care access, and reduce emergency department (ED) use but do not consistently improve clinical outcomes or reduce inpatient care use. ${ }^{16-19} \mathrm{~A}$ limitation of this literature is that studies have typically 
compared BHHs to no primary care, raising the question of whether BHHs are achieving comparable outcomes to usual primary care.

In contrast to the $\mathrm{BHH}$ model, in which $\mathrm{BH}$ organizations manage patients' care, the PCMH model tasks PCPs with addressing patients' health needs in collaboration with other medical providers. ${ }^{20}$ The PCMH model has been shown to improve some preventive screening and service use outcomes for people with SMI. ${ }^{21-24}$ However, Bowdoin et al. found that among people with mental illness, receiving care in a PCMH did not yield better preventive care or service use outcomes than having a non-PCMH usual source of care. ${ }^{25,}{ }^{26}$ Enhancements to the PCMH model designed to meet the complex health needs of people with SMI may better leverage the potential of the model to serve this population, but evidence regarding what these enhancements should be is lacking. ${ }^{27,} 28$

In this study, we examined a novel model of enhanced primary care for people with SMI. The enhanced primary care was delivered at WakeBrook Primary Care ("WakeBrook"), a primary care clinic co-located with aBH facility in Wake County, North Carolina. WakeBrook is a PCMH that provides care coordination, peer support, and self-management programs for people with SMI. ${ }^{15,28,29}$ Like BHHs, it is specifically geared toward the needs of people with SMI. The primary care services at WakeBrook tailored for individuals with SMI include the following: smaller patient panels, allowing providers to spend more time with each patient building trust; training for providers on working with people with SMI, to help providers understand the unique needs and challenges of this population and learn how to de-escalate crisis situations; and regular communication between PCPs and patients' behavioral health providers, enabling proactive planning to address patients' complex needs. ${ }^{30}$ WakeBrook receives referrals of people with SMI who are receiving outpatient behavioral healthcare from community providers but are not in primary care. As such, WakeBrook is generally not responsible for delivering regular outpatient psychiatric care to its patients, though patients can receive crisis and inpatient care at the co-located behavioral health facility. The objective of this study was to evaluate the effects of enhanced primary care, as compared to usual primary care, on healthcare use and screening among people with SMI. We focused specifically on patients with schizophrenia or schizoaffective disorder due to the difficulty of accurately identifying other types of SMI in administrative data.

\section{METHODS}

We used North Carolina Medicaid claims data to conduct a retrospective cohort study comparing healthcare use and screening receipt of people with SMI. We compared people with SMI newly receiving enhanced primary care to people with SMI newly receiving usual primary care. We constructed a comparison group of people with SMI newly receiving usual primary care in Mecklenburg County, a North Carolina county without an enhanced primary care clinic. We used inverse probability of treatment weighting to balance observed characteristics between the WakeBrook and comparison group. As sensitivity analyses, we compared the WakeBrook group to people with SMI from all North Carolina counties. All analyses were pre-specified.

\section{Data Sources}

We accessed North Carolina Medicaid claims and encounter data from January 2014 to September 2018 through the Carolina Cost and Quality Initiative. ${ }^{31}$ Approximately $18 \%$ of North Carolina's population is covered by Medicaid, which was not expanded under the Affordable Care Act. North Carolina's Medicaid program is currently a fee-for-service program but has a capitated BH carve-out wherein BH services are delivered by regional managed care organizations (MCOs). Our data included all claims from fee-for-service Medicaid and encounter data from the MCOs. We identified PCPs in the claims data by matching national provider identifiers (NPI) to the Centers for Medicare and Medicaid Services National Plan and Provider Enumeration System (NPPES), ${ }^{32}$ yielding a match rate of 98.2\%. The study was approved by the University of North Carolina at Chapel Hill Institutional Review Board.

\section{Setting and Participants}

While WakeBrook accepts patients with a broader range of mental illness diagnoses, we restricted our sample to adults with schizophrenia or schizoaffective disorder to ensure that our treatment and comparison groups had similar disease severity. Diagnoses of psychotic disorders in administrative data may be more accurate than other psychiatric diagnoses, including depression and anxiety disorders. ${ }^{33-35}$ Individuals were eligible for inclusion in the study if they were 18 years of age or older and received a diagnosis of schizophrenia or a schizoaffective disorder on at least two service claims on two separate days during the study period.

We focused on individuals newly receiving primary care services to identify the effect of receiving primary care on outcomes. We identified individuals who had a new primary care visit between April 2015 and March 2017 after at least 6 months without a primary care visit to increase the likelihood that our sample was comprised of new primary care patients. We defined primary care visits as visits with evaluation and management current procedural terminology (CPT) codes where the rendering provider was a primary care provider, defined using NPPES specialty taxonomy codes. The first observed primary care visit after 6 months without a primary care visit is referred to as the index visit. We required individuals to be enrolled in Medicaid for at least 8 of the 12 months before their index visit and for at least 14 of the 18 months after their index primary care visit in order to ensure a sufficient time window for observing health service use in the baseline and follow-up periods. 
The WakeBrook group consisted of individuals who had their index primary care visit at WakeBrook as identified by WakeBrook's billing NPI. We validated this group using electronic health records data. The primary comparison group consisted of individuals with schizophrenia or schizoaffective disorder with a new primary care visit who resided in Mecklenburg County, North Carolina. Mecklenburg was selected as a comparison county because it is a large, urban county similar to Wake County, where WakeBrook is located (Mecklenburg contains Charlotte, and Wake contains Raleigh), and it lacks an enhanced primary care clinic for people with SMI. The distance between Wake and Mecklenburg (approximately $150 \mathrm{mi}$ ) ensures individuals were likely not traveling between them for routine care. We also used a comparison group of adults with schizophrenia or schizoaffective disorder from any North Carolina county not attributed to WakeBrook to test the robustness of our findings.

\section{Outcome Measures}

We examined the health service use and recommended screening receipt by WakeBrook and comparison patients in the 18 months following their index primary care visit. Service use outcome measures included the total number of primary care visits, outpatient psychiatric visits, inpatient psychiatric admissions, inpatient psychiatric days, inpatient nonpsychiatric admissions, inpatient non-psychiatric days, and emergency department (ED) visits in the 18-month followup period. The 18-month follow-up period was selected as the longest follow-up period for which sufficient sample was available for analysis. We defined visit and admission types using CPT codes, provider specialty type from NPPES, place of service codes, and facility revenue codes. We defined psychiatric inpatient stays using diagnosis-related groups and revenue codes, and we classified non-psychiatric inpatient use as all other inpatient stays as defined by revenue codes. Screening outcome measures included binary indicators of receipt of the following screenings in the 18-month followup period: hemoglobin A1c, glucose, lipids, colorectal cancer, cervical cancer, and human immunodeficiency virus (HIV). We restricted to the relevant clinical subpopulation for each screening type, based on U.S. Preventive Services Task Force recommendations and the input of our study team's clinician members (Appendix Table 1). ${ }^{36}$

\section{Statistical Analysis}

We used inverse probability of treatment weighting (IPTW) with regression adjustment to balance the WakeBrook and comparison groups on factors that would affect the outcomes, including demographic characteristics, comorbid conditions, service use, screening receipt, and length of Medicaid enrollment in the 12 months prior to their index visit. We identified comorbid conditions following condition definitions from the Healthcare Cost and Utilization Project (HCUP) Clinical Classifications Software. ${ }^{37,38}$ The IPTW approach allowed us to mitigate selection bias due to observed differences between the WakeBrook and comparison groups. ${ }^{39}$

By comparing the absolute value of the standardized differences $(\times 100)$ in baseline characteristics between the WakeBrook and comparison group before and after weighting (Appendix Fig. 2), we concluded that we achieved appropriate balance between groups. ${ }^{40}$ We recalculated the weights to reflect the different sample compositions in the screening analyses restricted to relevant subpopulations and the sensitivity analyses using the alternate comparison group. We used linear models for all outcomes, assuming Gaussian error distributions. We present average marginal effects with $95 \%$ confidence intervals (CI) from delta-method standard errors. ${ }^{41}$

\section{RESULTS}

We identified 160 eligible individuals with an index enhanced primary care visit at WakeBrook, 1433 individuals with a usual primary care visit from Mecklenburg County, and 16,095 eligible individuals with an index primary care visit anywhere in North Carolina (see Fig. 1). The most prevalent comorbid conditions in the sample were substance-related disorders (68\% and $71 \%$ in the WakeBrook and Mecklenburg County groups, respectively), hypertension (52\% and 60\%), diabetes (49\% and 46\%), and hyperlipidemia (48\% and 58\%) (Table 1). We also observed high rates of comorbid bipolar disorder in both the WakeBrook and comparison groups (44\% and $40 \%$, respectively). Prior to weighting, the WakeBrook and comparison groups differed most dramatically with respect to the number of months of assertive community treatment (ACT) received in the 12 months before the index visit, number of psychiatric inpatient days in the 12 months before the index visit, proportion female, and proportion with congestive heart failure (Appendix Fig. 2).

Table 2 shows unadjusted monthly mean utilization measures in the 12 months before and 18 months after the index primary care visit, in order to make the baseline and follow-up periods comparable. The unadjusted monthly mean number of primary care visits in the WakeBrook group increased from 0.039 to $0.26(p<0.001)$ in the 12 months before and 18 months after the index visit (Table 2). WakeBrook patients had an unadjusted monthly mean of 0.20 primary care visits at WakeBrook in the 18 months after the index visit (not shown). The monthly mean values of all other service use measures did not significantly change with initiation of care at WakeBrook. Mecklenburg County comparison group patients experienced an increase from 0.047 to 0.23 in monthly mean primary care visits $(p<0.001)$. These patients also had increases in monthly mean outpatient psychiatric visits $(0.12$ to $0.13, p=0.011)$, non-psychiatric inpatient stays $(0.016$ to $0.026, p=0.0094)$, non-psychiatric inpatient days $(0.16$ to $0.28, p=0.020)$, and ED visits (0.21 to $0.24, p=0.0018)$.

In the adjusted analyses, we found that receiving enhanced primary care at WakeBrook was associated with an increase of 


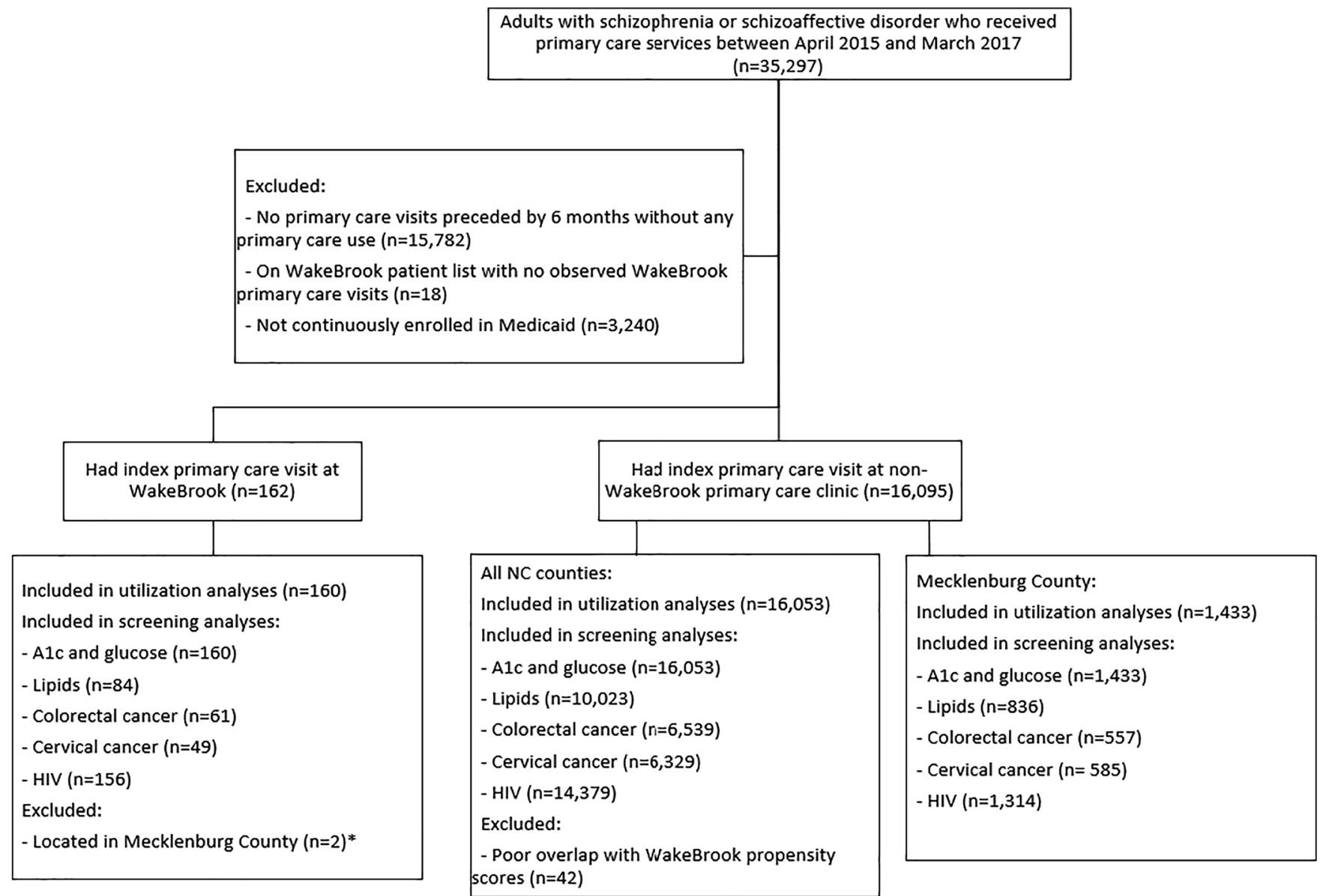

Figure 1 Construction of cohort of people with serious mental illness newly receiving primary care. The asterisk denotes participants not excluded from analyses with all NC counties comparison group. We excluded from the comparison groups 18 individuals who were on the list of WakeBrook patients based on the WakeBrook electronic health record list but who had no observed WakeBrook index primary care visit in Medicaid claims during our study period. We also excluded from the treatment group two individuals who had WakeBrook claims but whose recorded residence was in Mecklenburg County.

1.2 primary care visits ( $95 \% \mathrm{CI}: 0.31$ to 2.1 ) in the 18 months after the index visit compared to usual primary care (Table 3 ). Receiving enhanced primary care was associated with decreases of 0.33 non-psychiatric inpatient stays (CI: -0.49 to -0.16$)$ and 3.0 non-psychiatric inpatient days (CI: -5.3 to -0.60 ) in the follow-up period. Receipt of enhanced primary care had no significant effect on the number of outpatient psychiatric visits, psychiatric inpatient use, or ED visits.

Receiving enhanced primary care was associated with an increase of 8.8 percentage points (CI: 1.7 to 16 ) in the probability of receiving glucose screening in the 18 months after the index visit and an increase of 10 percentage points (CI: 3.7 to 17 ) in the probability of receiving HIV screening in the follow-up period compared to usual primary care. Receipt of enhanced primary care was associated with a decrease of 11 percentage points $(\mathrm{CI},-18$ to -3.3$)$ in the probability of receiving lipid screening in the follow-up period. Enhanced primary care had no significant effect on the probability of A1c and colorectal cancer screening receipt.

These results were robust when we included comparison group patients who had received primary care at any non-
WakeBrook location in North Carolina. In the service use analyses with this alternate comparison group, enhanced primary care was associated with decreases in the number of nonpsychiatric inpatient stays and days that were similar in magnitude to the Mecklenburg County analyses, but there was no longer a significant effect on primary care visits. We again observed increases in the probability of glucose and HIV screening, as well as an increase in the probability of A1c screening. We did not observe a decrease in the probability of lipid screening when using the full North Carolina comparison group.

\section{DISCUSSION}

We found that enhanced primary care led to some improvements in preventive screening over usual primary care, suggesting that enhanced primary care may be superior in quality to approaches that deliver traditional forms of primary care to people with SMI. Relative to the low baseline screening rates in our sample (e.g., $11 \%$ of comparison group patients 
Table 1 Baseline Characteristics of Individuals Who Newly Received Primary Care (Unweighted)

\begin{tabular}{|c|c|c|c|c|c|}
\hline & $\begin{array}{l}\text { Treatment } \\
\text { group } \\
(n=160)\end{array}$ & $\begin{array}{l}\text { Comparison group, } \\
\text { Mecklenburg County } \\
(n=1433)\end{array}$ & $\begin{array}{l}p \text { value, comparison } \\
\text { of treatment } \\
\text { and Mecklenburg } \\
\text { county comparison } \\
\text { groups } s\end{array}$ & $\begin{array}{l}\text { Comparison group } \\
\text { of non-WB patients, } \\
\text { all NC counties } \\
(n=16,053)\end{array}$ & $\begin{array}{l}p \text { value, comparison } \\
\text { of treatment and all } \\
\text { NC counties } \\
\text { comparison groups }\end{array}$ \\
\hline \multicolumn{6}{|c|}{ Demographic characteristics* } \\
\hline Age & $42 \pm 15$ & $45 \pm 15$ & 0.015 & $46 \pm 15$ & $<0.001$ \\
\hline Female & $33 \%$ & $48 \%$ & $<0.001$ & $48 \%$ & $<0.001$ \\
\hline \multicolumn{6}{|l|}{ Race } \\
\hline American Indian & $0.63 \%$ & $0.35 \%$ & 0.59 & $1.2 \%$ & 0.50 \\
\hline Asian & $1.9 \%$ & $2.2 \%$ & 0.81 & $0.66 \%$ & 0.065 \\
\hline Black & $64 \%$ & $70 \%$ & 0.12 & $52 \%$ & 0.0017 \\
\hline $\begin{array}{l}\text { More than one } \\
\text { race }\end{array}$ & $1.3 \%$ & $1.1 \%$ & 0.81 & $1.1 \%$ & 0.90 \\
\hline Race unknown & $4.4 \%$ & $5.0 \%$ & 0.75 & $4.2 \%$ & 0.93 \\
\hline White & $28 \%$ & $21 \%$ & 0.062 & $40 \%$ & $<0.001$ \\
\hline \multicolumn{6}{|l|}{ Ethnicity } \\
\hline Hispanic & $1.9 \%$ & $3.1 \%$ & 0.40 & $2.0 \%$ & 0.91 \\
\hline Not Hispanic/ & $91 \%$ & $83 \%$ & 0.0070 & $88 \%$ & 0.22 \\
\hline \multicolumn{6}{|l|}{ Latino } \\
\hline $\begin{array}{l}\text { Ethnicity } \\
\text { unknown }\end{array}$ & $6.9 \%$ & $14 \%$ & 0.012 & $9.7 \%$ & 0.21 \\
\hline \multicolumn{6}{|c|}{ Comorbid conditions } \\
\hline Diabetes & $49 \%$ & $46 \%$ & 0.46 & $44 \%$ & 0.26 \\
\hline Hypertension & $52 \%$ & $60 \%$ & 0.049 & $61 \%$ & 0.024 \\
\hline COPD & $21 \%$ & $28 \%$ & 0.070 & $30 \%$ & 0.027 \\
\hline Asthma & $22 \%$ & $23 \%$ & 0.67 & $23 \%$ & 0.89 \\
\hline Hyperlipidemia & $48 \%$ & $58 \%$ & 0.0090 & $51 \%$ & 0.44 \\
\hline $\begin{array}{l}\text { Alcohol-related } \\
\text { disorder }\end{array}$ & $24 \%$ & $31 \%$ & 0.085 & $23 \%$ & 0.59 \\
\hline $\begin{array}{l}\text { Substance- } \\
\text { related disorder }\end{array}$ & $68 \%$ & $71 \%$ & 0.50 & $63 \%$ & 0.15 \\
\hline $\begin{array}{l}\text { Acute } \\
\text { myocardial } \\
\text { infarction }\end{array}$ & $1.9 \%$ & $1.6 \%$ & 0.80 & $2.7 \%$ & 0.50 \\
\hline $\begin{array}{l}\text { Intarction } \\
\text { Coronary artery } \\
\text { disease }\end{array}$ & $6.9 \%$ & $11 \%$ & 0.15 & $13 \%$ & 0.027 \\
\hline $\begin{array}{l}\text { Congestive heart } \\
\text { failure }\end{array}$ & $1.3 \%$ & $8.7 \%$ & $<0.001$ & $9.6 \%$ & $<0.001$ \\
\hline HIV & $3.1 \%$ & $4.3 \%$ & 0.50 & $2.5 \%$ & 0.33 \\
\hline Hepatitis & $6.9 \%$ & $7.5 \%$ & 0.79 & $5.6 \%$ & 0.51 \\
\hline Bipolar disorder & $44 \%$ & $40 \%$ & 0.40 & $41 \%$ & 0.63 \\
\hline \multicolumn{6}{|c|}{ Baseline service receipt ${ }^{\dagger}$} \\
\hline $\begin{array}{l}\text { Months of ACT } \\
\text { receipt }\end{array}$ & $3.8 \pm 5.0$ & $0.93 \pm 2.9$ & $<0.001$ & $1.0 \pm 3.0$ & $<0.001$ \\
\hline \multicolumn{6}{|l|}{$\begin{array}{l}\text { Medicare- } \\
\text { eligible }\end{array}$} \\
\hline $\begin{array}{l}\text { Ever received } \\
\text { SSI }\end{array}$ & $78 \%$ & $70 \%$ & 0.026 & $69 \%$ & 0.014 \\
\hline Months & $12 \pm 0.94$ & $12 \pm 0.68$ & 0.041 & $12 \pm 0.62$ & 0.0023 \\
\hline \multicolumn{6}{|c|}{$\begin{array}{l}\text { Medicaid } \\
\text { enrolled }\end{array}$} \\
\hline \multicolumn{6}{|c|}{ Screening receipt in 12 months before index visit } \\
\hline Alc & $19 \%$ & $19 \%$ & 0.92 & $14 \%$ & 0.086 \\
\hline Glucose & $13 \%$ & $11 \%$ & 0.49 & $9.1 \%$ & 0.093 \\
\hline Lipids & $14 \%$ & $25 \%$ & 0.035 & $18 \%$ & 0.33 \\
\hline \multicolumn{5}{|l|}{ cancer } & 0.94 \\
\hline Cervical cancer & $8.2 \%$ & $11 \%$ & 0.52 & $11 \%$ & 0.85 \\
\hline HIV & $7.7 \%$ & $12 \%$ & 0.10 & $9.1 \%$ & 0.70 \\
\hline
\end{tabular}

COPD chronic obstructive pulmonary disease, HIV human immunodeficiency virus, ACT assertive community treatment, SSI Supplemental Security Income

*Continuous variables reported as means with standard deviation and binary variables as percentages

${ }^{\dagger}$ Months of ACT receipt and Medicaid enrollment observed in 12 months prior to index primary care visit. Medicare dual eligibility and receipt of SSI observed across the study period

${ }^{*}$ Refers to percentage that received screening type in the 12 months prior to index primary care visit, restricted to the relevant subpopulation for the screening.

$s_{p}$ values are from t tests of means of continuous variables and Pearson's chi-squared tests of categorical variables

received glucose screening in the 12 months prior to treatment and $12 \%$ received HIV screening), the magnitude of the increases in screening probability we observed was substantial. The two exceptions to this were lipid and colorectal cancer screening. The relatively short follow-up period of 18 months used here may not allow for enough time to build 
Table 2 Unadjusted Monthly Mean Utilization 12 Months Before and 18 Months After Index Primary Care Visit by Treatment Status

\begin{tabular}{llll}
\hline \hline & Pre-treatment monthly mean & Post-treatment monthly mean & $p$ value, comparison of means* \\
\hline Treatment group & & & $<0.001$ \\
Primary care visits & 0.039 & 0.26 & 0.79 \\
Outpatient psychiatric visits & 0.15 & 0.16 & 0.37 \\
Psychiatric inpatient stays & 0.031 & 0.025 & 0.70 \\
Psychiatric inpatient days & 0.46 & 0.0063 & 0.72 \\
Non-psychiatric inpatient stays & 0.0073 & 0.068 & 0.70 \\
Non-psychiatric inpatient days & 0.054 & 0.22 & 0.96 \\
ED visits & 0.22 & 0.23 & $<0.001$ \\
Comparison group, Mecklenburg County & 0.047 & 0.13 & 0.011 \\
Primary care visits & 0.12 & 0.020 & 1.0 \\
Outpatient psychiatric visits & 0.020 & 0.24 & 0.12 \\
Psychiatric inpatient stays & 0.20 & 0.026 & 0.0094 \\
Psychiatric inpatient days & 0.016 & 0.28 & 0.020 \\
Non-psychiatric inpatient stays & 0.16 & & 0.0018 \\
Non-psychiatric inpatient days & 0.21 & 0.25 & $<0.001$ \\
ED visits & & 0.13 & $<0.001$ \\
Comparison group, all NC counties & 0.046 & 0.015 & 0.58 \\
Primary care visits & 0.098 & 0.16 & 0.42 \\
Outpatient psychiatric visits & 0.015 & 0.029 & $<0.001$ \\
Psychiatric inpatient stays & 0.16 & 0.31 & $<0.001$ \\
Psychiatric inpatient days & 0.020 & & $<0.001$ \\
Non-psychiatric inpatient stays & 0.22 & 0.18 &
\end{tabular}

*t test of means pre- and post-treatment

trust for invasive procedures like colonoscopy. The data used here also does not allow us to assess whether there are clinically appropriate reasons for these screening rates to differ.

We also found that enhanced primary care for people with SMI resulted in fewer non-psychiatric inpatient hospitalizations and more primary care visits compared to usual primary care. Per our estimates, for every ten people with SMI newly enrolled in enhanced primary care rather than usual primary care, three non-psychiatric inpatient stays could be avoided and twelve additional primary care visits would take place over 18 months. Enhanced primary care may have increased patients' engagement in primary care and better controlled patients' physical health conditions compared to usual primary care, resulting in less need for inpatient care. Effects on psychiatric inpatient stays and days were not statistically significant and centered near zero, which could be due to WakeBrook patients' proximity to a $\mathrm{BH}$ inpatient facility resulting in greater likelihood of hospitalization for $\mathrm{BH}$ reasons. Effects on ED visits were negative but not statistically significant.

Our results suggest that reductions in inpatient use and improvements in healthcare quality may be achievable from adapting primary care services for people with SMI. A potential advantage of the enhanced primary care model is that it could be adopted by existing PCMHs to provide integrated care for people with SMI, especially when BHHs are not present. However, unlike most PCMHs, WakeBrook is colocated within a behavioral health facility that offers crisis and inpatient psychiatric services. Implementing enhanced primary care in other contexts may require adaptations to offer primary care providers additional support. Primary care practices' ability to incorporate these components would likely rely on enhanced or flexible payment models. The use of claims data in our study introduces limitations, like the inability to observe clinical outcomes and the potential lack of reliability of diagnostic information. We notably found very high rates of comorbid bipolar disorder in our patient samples, which may result from misdiagnoses. This study was only focused on North Carolina Medicaid enrollees and services reimbursed by Medicaid; therefore, we could not observe healthcare services provided by other primary care providers outside of Medicaid or North Carolina. Our analysis was limited to patients with psychotic disorders newly enrolled in primary care, as defined by a 6-month washout period, so our results are generalizable to that population. In addition, if any comparison practices offered primary care tailored for adults with SMI, our estimates would be overly conservative. Our analyses also have several strengths. We used IPTW with regression adjustment to control for selection bias. We controlled for several important baseline characteristics, including individuals' service use and screening receipt prior to newly receiving primary care services, improving comparability of our patient groups.

Overall, our study suggests that enhanced primary care for people with SMI shows promise. Future research should study the effects of enhanced primary care when implemented across different settings and populations and identify the specific intervention components that affect outcomes. In a 2017 study examining a PCMH model enhanced for high-cost, high-need populations in VA settings, Zulman et al. found that this enhanced primary care did not reduce acute care use compared to usual primary care, suggesting that enhanced care may require specific features to be effective. ${ }^{42}$ Understanding which intervention components are effective in which contexts would allow health system administrators and policymakers to 
Table 3 Unadjusted Utilization and Screening Receipt 18 Months Post-treatment and IPTW Average Treatment Effects of Receiving Primary Care at WakeBrook on Utilization and Screening Receipt

\begin{tabular}{|c|c|c|c|}
\hline Outcome & Treatment group ${ }^{\S}$ & Comparison group & $\begin{array}{l}\text { Average treatment effect } \\
\text { (95\% confidence interval) }\end{array}$ \\
\hline \multirow{2}{*}{\multicolumn{4}{|c|}{ Mecklenburg County comparison }} \\
\hline & & & Utilization $^{\dagger}$ \\
\hline Primary care visits & $4.7 \pm 5.1$ & $4.2 \pm 4.4$ & $1.2 *(0.31,2.1)$ \\
\hline Outpatient psychiatric visits & $2.8 \pm 5.2$ & $2.4 \pm 3.6$ & $-0.18(-0.73,0.38)$ \\
\hline Psychiatric inpatient stays & $0.44 \pm 0.97$ & $0.35 \pm 1.1$ & $-0.024(-0.16,0.12)$ \\
\hline Psychiatric inpatient days & $7.4 \pm 18$ & $4.3 \pm 17$ & $-0.013(-2.5,2.5)$ \\
\hline Non-psychiatric inpatient stays & $0.11 \pm 0.48$ & $0.48 \pm 2.7$ & $-0.33 *(-0.49,-0.16)$ \\
\hline Non-psychiatric inpatient days & $1.2 \pm 7.4$ & $5.0 \pm 33$ & $-3.0 *(-5.3,-0.60)$ \\
\hline ED visits & $4.0 \pm 8.6$ & $4.3 \pm 7.7$ & $-0.80(-1.8,0.18)$ \\
\hline \multicolumn{4}{|l|}{ Screening receipt ${ }^{\ddagger}$} \\
\hline A1c screening & $37 \%$ & $35 \%$ & $-0.013(-0.094,0.068)$ \\
\hline Glucose screening & $21 \%$ & $19 \%$ & $0.088 *(0.017,0.16)$ \\
\hline Lipids screening & $30 \%$ & $42 \%$ & $-0.11(-0.18,-0.033) *$ \\
\hline Colorectal cancer screening & $21 \%$ & $11 \%$ & $0.063(-0.064,0.19)$ \\
\hline HIV screening & $25 \%$ & $18 \%$ & $0.10 *(0.037,0.17)$ \\
\hline \multicolumn{4}{|l|}{ All NC counties comparison" } \\
\hline \multicolumn{4}{|l|}{ Utilization $^{\dagger}$} \\
\hline Primary care visits & $4.7 \pm 5.1$ & $4.6 \pm 5.1$ & $0.76(-0.37,1.9)$ \\
\hline Outpatient psychiatric visits & $2.8 \pm 5.2$ & $2.4 \pm 4.0$ & $0.77(-0.12,1.7)$ \\
\hline Psychiatric inpatient stays & $0.45 \pm 1.0$ & $0.27 \pm 0.91$ & $0.074(-0.059,0.21)$ \\
\hline Psychiatric inpatient days & $7.9 \pm 19$ & $2.9 \pm 12$ & $6.1(-0.0033,12.1)$ \\
\hline Non-psychiatric inpatient stays & $0.11 \pm 0.47$ & $0.52 \pm 3.2$ & $-0.41 *(-0.59,-0.24)$ \\
\hline Non-psychiatric inpatient days & $1.2 \pm 7.4$ & $5.6 \pm 37$ & $-3.9 *(-6.7,-1.1)$ \\
\hline ED visits & $4.0 \pm 8.5$ & $3.7 \pm 7.3$ & $-0.29(-1.6,1.0)$ \\
\hline \multicolumn{4}{|l|}{ Screening receipt ${ }^{\ddagger}$} \\
\hline A1c screening & $37 \%$ & $27 \%$ & $0.10 *(0.0051,0.20)$ \\
\hline Glucose screening & $21 \%$ & $15 \%$ & $0.084 *(0.012,0.16)$ \\
\hline Lipids screening & $29 \%$ & $32 \%$ & $-0.0036(-0.066,0.058)$ \\
\hline Colorectal cancer screening & $21 \%$ & $14 \%$ & $0.045(-0.058,0.15)$ \\
\hline HIV screening & $25 \%$ & $15 \%$ & $0.11 *(0.039,0.18)$ \\
\hline
\end{tabular}

$* p<0.05$

All utilization outcomes refer to total number of visits or inpatient stay days across the 18-month follow-up period

${ }^{7}$ All screening outcomes refer to whether the screening type was received in the 18-month follow-up period

${ }^{s}$ Continuous variables reported as means with standard deviation and binary variables as percentages

"The treatment group in these analyses included two additional individuals who were excluded from the Mecklenburg County comparison group analyses because they resided in Mecklenburg County

better determine whether and how to invest in enhanced primary care for people with SMI.

Supplementary Information The online version contains supplementary material available at https://doi.org/10.1007/s11606-02006429-2.

Corresponding Author: Lexie R. Grove, MSPH; Department of Health Policy and Management, Gillings School of Global Public Health, University of North Carolina at Chapel Hill, 135 Dauer Dr., Chapel Hill, NC 27599-7411, USA (e-mail: lrgrove@live.unc.edu).

Funding This research was funded by North Carolina Translational and Clinical Sciences (NC TraCS, National Institutes of Health (NIH) CTSA No. UL1TRO02489) Grant No. 550KR171716. The database infrastructure used for this project was supported by the Cecil G. Sheps Center for Health Services Research and the CER Strategic Initiative of UNC's Clinical and Translational Science Award (UL1TR001111). This research was partially supported by a National Research Service Award Pre-Doctoral Traineeship from the Agency for Healthcare Research and Quality sponsored by The Cecil G. Sheps Center for Health Services Research, The University of North Carolina at Chapel Hill, Grant No. T32-HSOOOO32. The intervention described in this study was funded by SAMHSA Primary and Behavioral Health Care Integration Grant No. SM-15-005.
Conflict of Interest: The authors declare that they do not have a conflict of interest.

\section{REFERENCES}

1. Substance Abuse and Mental Health Service Administration. Key substance use and mental health indicators in the United States: Results from the 2018 National Survey on Drug Use and Health (HHS Publication No. PEP19-5068, NSDUH Series H-54). Rockville, MD: Center for Behavioral Health Statistics and Quality, Substance Abuse and Mental Health Service Administration; 2019. Available from: https://www. samhsa.gov/data/sites/default/files/cbhsq-reports/NSDUHNationalFindingsReport2018/NSDUHNationalFindingsReport2018.pdf.

2. Federal Register. 1993;58(NO. 96):29422-5.

3. De Hert M, Correll CU, Bobes J, Cetkovich-Bakmas M, Cohen D, Asai I, et al. Physical illness in patients with severe mental disorders. I. Prevalence, impact of medications and disparities in health care. World Psychiatry 2011;10(1):52-77.

4. Lin WC, Zhang J, Leung GY, Clark RE. Chronic physical conditions in older adults with mental illness and/or substance use disorders. J Am Geriatr Soc 2011;59(10): 1913-21.

5. Jones DR, Macias C, Barreira PJ, Fisher WH, Hargreaves WA, Harding CM. Prevalence, severity, and co-occurrence of chronic physical health problems of persons with serious mental illness. Psychiatr Serv 2004;55(11): 1250-7.

6. Hansen RA, Voils CI, Farley JF, Powers BJ, Sanders LL, Sleath B, et al. Prescriber continuity and medication adherence for complex patients. Ann Pharmacother 2015;49(3):293-302. 
7. Wong MC, Liu J, Zhou S, Li S, Su X, Wang HH, et al. The association between multimorbidity and poor adherence with cardiovascular medications. Int J Cardiol 2014; 177(2):477-82.

8. O'Shea MP, Teeling M, Bennett $\mathbf{K}$. An observational study examining the effect of comorbidity on the rates of persistence and adherence to newly initiated oral anti-hyperglycaemic agents. Pharmacoepidemiol Drug Saf 2013;22(12): 1336-44.

9. Domino ME, Beadles CA, Lichstein JC, Farley JF, Morrissey JP, Ellis AR, et al. Heterogeneity in the quality of care for patients with multiple chronic conditions by psychiatric comorbidity. Med Care 2014;52:S101-S9.

10. Dickerson FB, Goldberg RW, Brown CH, Kreyenbuhl JA, Wohlheiter $\mathbf{K}$, Fang $\mathbf{L}$, et al. Diabetes knowledge among persons with serious mental illness and type 2 diabetes. Psychosomatics. 2005;46(5):418-24.

11. Egede LE. Major depression in individuals with chronic medical disorders: prevalence, correlates and association with health resource utilization, lost productivity and functional disability. Gen Hosp Psychiatry 2007;29(5):409-16. https://doi.org/10.1016/j.genhosppsych.2007. 06.002 .

12. Stein MB, Cox BJ, Afifi TO, Belik S-L, Sareen J. Does co-morbid depressive illness magnify the impact of chronic physical illness? A population-based perspective. Psychol Med 2006;36(5):587-96.

13. Katon WJ. Clinical and health services relationships between major depression, depressive symptoms, and general medical illness. Biol Psychiatry 2003;54(3):216-26.

14. Parks J, Svendsen D, Singer P, Foti ME, Mauer B. Morbidity and mortality in people with serious mental illness. Alexandria, VA: National Association of State Mental Health Program Directors (NASMHPD) Medical Directors Council. 2006;25(4):1-87.

15. Center for Integrated Health Solutions. Behavioral health homes for people with mental health \& substance use conditions: the core clinical features. Washington, DC;2012. Available from: www.integration.samhsa.gov.

16. Fortuna KL, DiMilia PR, Lohman MC, Cotton BP, Cummings JR, Bartels SJ, et al. Systematic Review of the Impact of Behavioral Health Homes on Cardiometabolic Risk Factors for Adults With Serious Mental Illness. Psychiatr Serv. 2019:appi. ps. 201800563.

17. Murphy KA, Daumit GL, Stone E, McGinty EE. Physical health outcomes and implementation of behavioural health homes: a comprehensive review. Int Rev Psychiatry 2018;30(6):224-41.

18. Bandara SN, Kennedy-Hendricks A, Stuart EA, Barry CL, Abrams MT, Daumit GL, et al. The effects of the Maryland Medicaid Health Home Waiver on Emergency Department and inpatient utilization among individuals with serious mental illness. Gen Hosp Psychiatry 2020;64:99-104.

19. Murphy KA, Daumit GL, Bandara SN, Stone EM, Kennedy-Hendricks A, Stuart EA, et al. Association Between the Maryland Medicaid Behavioral Health Home Program and Cancer Screening in People With Serious Mental Illness. Psychiatr Serv 2020;71(6):608-11.

20. Agency for Healthcare Research and Quality. Defining the PCMH. Available from: https://pcmh.ahrq.gov/page/defining-pcmh.

21. Domino ME, Wells R, Morrissey JP. Serving Persons With Severe Mental Illness in Primary Care-Based Medical Homes. Psychiatr Serv 2015;66(5):477-83. https://doi.org/10.1176/appi.ps.201300546.

22. Grove LR, Olesiuk WJ, Ellis AR, Lichstein JC, DuBard CA, Farley JF, et al. Evaluating the potential for primary care to serve as a mental health home for people with schizophrenia. Gen Hosp Psychiatry 2017;47:14-9.

23. Grove LR, Domino ME, Farley JF, Swietek KE, Beadles C, Ellis AR, et al. Medical Home Effects on Enrollees With Mental and Physical Illness. Am J Manag Care 2020;26(5):218-23.

24. Domino ME, Kilany M, Wells R, Morrissey JP. Through the looking glass: estimating effects of medical homes for people with severe mental illness. Health Serv Res 2017;52(5):1858-80.
25. Bowdoin JJ, Rodriguez-Monguio R, Puleo E, Keller D, Roche J. Associations between the patient-centered medical home and preventive care and healthcare quality for non-elderly adults with mental illness: A surveillance study analysis. BMC Health Serv Res 2016;16(1):434.

26. Bowdoin JJ, Rodriguez-Monguio R, Puleo E, Keller D, Roche J. The patient-centered medical home model: healthcare services utilization and cost for non-elderly adults with mental illness. J Ment Health 2018;27(6):574-82.

27. O'Toole TP, Pirraglia P, Dosa D, Bourgault C, Redihan S, O'Toole M, et al. Building care systems to improve access for high-risk and vulnerable veteran populations. J Gen Intern Med 2011;26(2):683.

28. Alakeson V, Frank RG, Katz RE. Specialty care medical homes for people with severe, persistent mental disorders. Health Aff 2010;29(5):867-73.

29. Croghan TW, Brown JD. Integrating mental health treatment into the patient centered medical home. Rockville: Agency for Healthcare Research and Quality, 2010.

30. Perrin J, Reimann B, Capobianco J, Wahrenberger JT, Sheitman BB, Steiner BD. A model of enhanced primary care for patients with severe mental illness. N C Med J 2018;79(4):240-4.

31. The Carolina Cost and Quality Initiative. 2019. Available from: https:// www.shepscenter.unc.edu/data/bcbsnc-claims-data-ccqi/.

32. Centers for Medicare and Medicaid Services. Data dissemination. 2018. Available from: https://www.cms.gov/Regulations-and-Guidance/Administrative-Simplification/NationalProvIdentStand/DataDissemination. html.

33. Walkup JT, Boyer CA, Kellermann SL. Reliability of Medicaid claims files for use in psychiatric diagnoses and service delivery. Adm Policy Ment Health Ment Health Serv Res 2000;27(3):129-39.

34. Spettell CM, Wall TC, Allison J, Calhoun J, Kobylinski R, Fargason R, et al. Identifying physician-recognized depression from administrative data: Consequences for quality measurement. Health Serv Res 2003;38(4): 1081-102.

35. Davis KA, Sudlow CL, Hotopf M. Can mental health diagnoses in administrative data be used for research? A systematic review of the accuracy of routinely collected diagnoses. BMC Psychiatry 2016;16(1):1-11.

36. U.S. Preventive Services Task Force. A and B recommendations. Available from: https://www.uspreventiveservicestaskforce.org/uspstf/recommendation-topics/uspstf-and-b-recommendations.

37. Healthcare Cost and Utilization Project. Clinical Classifications Software (CCS) for ICD-9-CM. Available from: https://www.hcup-us.ahrq.gov/ toolssoftware/ccs/ccs.jsp.

38. Healthcare Cost and Utilization Project. Clinical Classifications Software (CCS) for ICD-10-PCS (beta version). Available from: https://www.hcupus.ahrq.gov/toolssoftware/ccs10/ccs10.jsp.

39. Austin PC. An introduction to propensity score methods for reducing the effects of confounding in observational studies. Multivar Behav Res 2011;46(3):399-424

40. Austin PC. Balance diagnostics for comparing the distribution of baseline covariates between treatment groups in propensity-score matched samples. Stat Med 2009;28(25):3083-107.

41. Oehlert GW. A note on the delta method. Am Stat 1992;46(1):27-9.

42. Zulman DM, Chee CP, Ezeji-Okoye SC, Shaw JG, Holmes TH, Kahn JS, et al. Effect of an intensive outpatient program to augment primary care for high-need Veterans Affairs patients: a randomized clinical trial. JAMA Intern Med 2017;177(2):166-75

Publisher's Note: Springer Nature remains neutral with regard to jurisdictional claims in published maps and institutional affiliations. 